\section{Breastfeeding and systemic agents for psoriasis}

In Tso et al's article, it is stated that females on adalimumab should not breastfeed until at least 5 months after the last treatment, according to the manufacturer's information. ${ }^{1}$ However, the majority of manufacturers have recently updated their information and now state that adalimumab can be used during breastfeeding.

The NICE guideline PH11 Maternal and Child Nutrition ${ }^{2}$ recommends that supplementary sources of information should be consulted regarding the prescribing of drugs to breastfeeding mothers, and that the BNF should only be used as a 'guide'.

The BNF contains little quantitative data on which to make informed decisions. The Summary of Product Characteristics of the vast majority of drugs recommends that they are not used during lactation. This does not imply risk, more that the manufacturers are not required to take responsibility. ${ }^{3}$

I consulted the UK Drugs in Lactation Advisory Service (UKDILAS - https://www. sps.nhs.uk/articles/ukdilas// and Lactmed (https://toxnet.nlm.nih.gov/pda/lactmed. $\mathrm{htm})$, both specialist sources of information for prescribing in lactation. Lactmed states:

Limited information indicates that maternal adalimumab injections produce low levels in breastmilk and do not adversely affect the nursing infant. Because adalimumab is a large protein molecule ... absorption is unlikely because it is probably destroyed in the infant's gastrointestinal tract. Most experts feel that the drug is probably safe during nursing. However, until more data become available, adalimumab should be used with caution while nursing a newborn or preterm infant.'

Although there is no published information on the use of secukinumab during breastfeeding, as it is a large protein molecule, the amount in milk is likely to be very low. Absorption is unlikely as it is probably destroyed in the infant's gastrointestinal tract. As for adalimumab, until more data become available, secukinumab should be used with caution during breastfeeding, especially while nursing a newborn or preterm infant.

UKDILAS concludes that the benefits of breastfeeding on adalimumab and secukinumab outweigh the risks.

As breastfeeding is of proven benefit to both mother and baby, withholding breastfeeding should not be considered a 'no-harm' option. It is important that breastfeeding mothers are given informed choice with access to balanced information.

Sarah Little,

GP, Dedridge Medical Group, Livingston.

Email: sarahealittleahotmail.com

Sarah Fenner,

West Midlands Medicines Information Service \& UK Drugs in Lactation Advisory Service.

\section{REFERENCES}

1. Tso S, Hunt W, Frow H, Ilchyshyn A. Systemic agents for psoriasis and their relevance to primary care. Br J Gen Pract 2019; DOI: https://doi. org/10.3399/bjgp19X701225.

2. National Institute for Health and Care Excellence. Maternal and child nutrition. PH11. 2008. https:// unw.nice.org.uk/guidance/ph11 laccessed $4 \mathrm{Mar}$ 2019).

3. Jones W, GP Infant Feeding Network. Safety of drugs in breastmilk. 2017. https://gpifn.org.uk/ drugs-in-breastmilk laccessed 4 Mar 2019).

DOI: https://doi.org/10.3399/bjgp19X702149

\section{Genetic cancer risk} assessment in general practice: systematic review of tools available, clinician attitudes, and patient outcomes

The systematic review by Laforest et al is a timely addition to understand the challenges that expanding genetic risk assessment and into primary care pose. ${ }^{1}$ This is especially opportune given its online publication in the same week that the Secretary of State Matt Hancock announced the plan for the NHS to offer genomic testing to healthy individuals for a fee. ${ }^{2}$

Direct-to-consumer genetic testing is already available. Companies such as 23andme (https://www.23andme.com/ en-gb/dna-health-ancestry/\#all-reportslist) will give you a 'genetic health risk' including the cancer risk genes (BRCA1 and 2), Alzheimer's dementia (APOE variant), as well as several other later-onset conditions and carrier status for in excess of 40 recessive conditions. Such testing currently has significant limitations, increasing health anxiety for some and falsely reassuring others. ${ }^{3}$ Such tests include substantial disclaimers, advice to discuss findings with healthcare professionals, and having confirmatory testing before taking action on any findings.

The NHS is also 'mainstreaming' genomic technologies for its patients, with the adoption of genetic testing outside of its traditional domain, clinical genetic departments. This change requires increased genomic literacy - discussing risks, interpreting results, and managing uncertainty - across a range of healthcare professionals including GPs.

The review by Laforest et al highlights the lack of knowledge and confidence that GPs have in one of these key areas, cancer genetics. ${ }^{1}$ They highlight the uncertainties and inconsistencies in how one should approach such patients and the lack of capacity in primary care to take on such a role. With the NHS now endorsing such direct-to-consumer testing, patients will undoubtedly wish and expect to be able to discuss findings with their GPs, who currently are inadequately prepared and resourced to do such a job.

How one should approach such a challenge is unclear. There is certainly a need for greater genomics education for the primary care team. It may also require a restructuring or expansion of the clinical genetics services, even a role for primary care-based genetic services. ${ }^{4}$ Greater clarity of appropriate referral pathways and respective responsibilities will also be critical to ensure that appropriate advice is given and resources are used optimally.

This review highlights some of the issues that will need to be overcome to fully 
embrace the potential of these genomic technologies in primary care.

\section{Will RH Evans,}

GP and NIHR In-Practice Fellow, Division of Primary Care, NIHR School for Primary Care Research, University of Nottingham, Nottingham.

Email: William.Evans1anottingham.ac.uk

\section{REFERENCES}

1. Laforest F, Kirkegaard P, Mann B, Edwards A. Genetic cancer risk assessment in general practice: systematic review of tools available, clinician attitudes, and patient outcomes. $\mathrm{Br} J$ Gen Pract 2019; DOI: https://doi.org/10.3399/ bjgp18X700265.

2. NHS to offer paid-for DNA tests if patients share data. BBC News 2019: 26 Jan: https://www.bbc. co.uk/news/uk-47013914 laccessed 5 Mar 2019].

3. Rafi I, Qureshi N, Lucassen A, et al. 'Over-thecounter' genetic testing: what does it really mean for primary care? Br J Gen Pract 2009; DOI: https://doi.org/10.3399/bjgp09X395021.

4. Westwood G, Pickering R, Latter S, et al. A primary care specialist genetics service: a clusterrandomised factorial trial. Br J Gen Pract 2012: DOI: https://doi.org/10.3399/bjgp12X630089

\section{Faecal}

\section{immunochemical (rule-in) testing in general practice}

D'Souza and colleagues underestimate GPs' clinical judgement in selecting patients for a faecal immunochemical test (FIT). ${ }^{1}$ NICE may recommend FIT for 'lowrisk' symptomatic patients 'without rectal bleeding who have unexplained labdominall symptoms but do not meet the criteria for a suspected cancer', ${ }^{2}$ but this has not led to the 'deluge' of referrals or worsening of the 'endoscopy capacity crisis' in the centres where FIT has been adopted. ${ }^{3}$

The majority of the estimated $10 \%$ of consulting patients with abdominal complaints will not be referred for colonoscopy. ${ }^{1}$ GPs conduct a careful triage using history and examination, an understanding of their patients' consulting patterns and comorbidity, preferences for testing, and by deciding when to respond to a positive result. Only a highly selected group of those tested and with a positive FIT are referred.

The NICE positive predictive value (PPV) threshold to rule in patients for urgent referral is 3\%: the PPV for a low-risk symptom such as abdominal pain is $2 \%$ (increasing with age) compared with 5\% for rectal bleeding. ${ }^{4}$ The PPV of a positive FIT in the low-risk symptomatic population is estimated at $13 \% .^{5}$ If FIT is positive, referral is uncontroversial; if negative, the PPV falls to $<1 \%$, making colonoscopy non-referral reasonable. FIT is more likely to result in a reduction of unnecessary (routine) endoscopy referrals for low-risk symptoms

The UK's routes to diagnosis data, cited by D'Souza, show us that a higher proportion of cancers are diagnosed at early stage when GPs investigate patients who do not meet 2WW criteria: those eligible for FIT. In order to achieve our nationwide target of 3 in 4 cancers diagnosed at an early stage by $2028,{ }^{6}$ offering early investigation land where necessary, referral) to the correct patients is crucial before more serious symptoms develop.

As a rule-in test for patients with low-risk symptoms, FIT enables the timely detection of cancer and other bowel disease in primary care. It may also play a role as a rule-out test for patients with highrisk symptoms, or in the future replace routine post-polypectomy colonoscopy surveillance. Introducing FIT may have been the most important change in the whole of NG12.4

\section{Sarah ER Bailey,}

Research Fellow, College of Medicine and Health, University of Exeter, Exeter. Email: S.E.R.Baileyldexeter.ac.uk

\section{Marije A van Melle,}

Research Associate, Department of Public Health and Primary Care, University of Cambridge, Cambridge.

\section{Brian D Nicholson,}

GP Clinical Researcher, Nuffield

Department of Primary Care Health

Sciences, University of Oxford, Oxford.

\section{REFERENCES}

1. D’Souza N, Brzezicki A, Abulafi M. Faecal immunochemical testing in general practice. $\mathrm{Br}$ J Gen Pract 2019; DOI: https://doi.org/10.3399/ bjgp19X700853.

2. National Institute for Health and Care Excellence.
Quantitative faecal immunochemical tests to guide referral for colorectal cancer in primary care. DG30. 2017. https://www.nice.org.uk/guidance/ dg30/chapter/1-Recommendations laccessed 3 Mar 2019].

3. Nicholson BD, James T, East JE, et al. Experience of adopting faecal immunochemical testing to meet the NICE colorectal cancer referral criteria for low-risk symptomatic primary care patients in Oxfordshire, UK. Frontline Gastroenterol 2018; DOI: 10.1136/flgastro-2018-101052.

4. National Institute for Health and Care Excellence. Suspected cancer: recognition and referral. NG12. 2015. http://www.nice.org.uk/guidance/NG12 laccessed 3 Mar 2019).

5. Juul JS, Hornung N, Andersen B, et al. The value of using the faecal immunochemical test in general practice on patients presenting with nonalarm symptoms of colorectal cancer. Br J Cancer 2018; DOI: http://dx.doi.org/10.1038/s41416-0180178-7

6. Alderwick H, Dixon J. The NHS long term plan. BMJ 2019; 364: 184

DOI: https://doi.org/10.3399/bjgp19X702173

\section{Correction}

In the Editorial by Nicholson BD, Perera R, and Thompson MJ. The elusive diagnosis of cancer: testing times. Br J Gen Pract 2018; DOI: https:// doi.org/10.3399/bjgp18X699461, Rafael Perera's affiliation and funding information was incomplete. The affiliation should have been: Rafael Perera, Professor of Medical Statistics, Nuffield Department of Primary Care Health Sciences, University of Oxford; NIHR Oxford Biomedical Research Centre, Oxford University Hospitals NHS Foundation Trust Oxford, UK. Additional funding information is: Rafael Perera is supported by the NIHR Oxford Biomedical Research Centre.

DOI: https://doi.org/10.3399/bjgp19X702185 